\title{
Fonctions et îlots textuels des passages guillemetés dans Le confident
}

\author{
Dr. Perihane Adel \\ Maitre de conférences -Université de Helwan- Egypte
}

\section{Résumé :}

Les guillemets constituent un outil important au niveau scriptural. Loin d'être un simple signe typographique, les guillemets ont plusieurs rôles dans les textes littéraires. A travers notre œuvre romanesque Le confident, premier roman d'Hélène Grémillon, nous procédons à l'analyse des fonctions et des îlots textuels des passages guillemetés.

\section{Abstract :}

The quotes are an important scriptural level tool. Far from being a simple typographical sign, quotation marks have many roles in literary texts. Through our novel the confident, first novel of Hélène Grémillon, we analyses autonymic functions and textual block of quoted passages.

Mots clés : Guillemets - fonction autonymique - îlot textuel - locuteur.

Keywords : Quotes - autonymic function - textual block - speaker

\section{Introduction}

Les guillemets constituent un champ d'étude riche qui a attiré l'attention d'un certain nombre de chercheurs. D'après le Petit Robert (2011), ils sont un «signe typographique qu'on emploie par paires («...») pour isoler un mot, un groupe de mots, etc., cités ou rapportés, pour indiquer un sens, pour se distancer d'un emploi ou pour mettre en valeur ». Pour J.Authier-Revuz, elle pose « le guillemet » comme un signe « de langue écrite à part entière », qui indique un commentaire sur l'emploi du dire requérant ainsi le statut d'un « signe sur les signes» (Authier-Revuz, 1998 : 373). Quant à G.Mourad, les guillemets constituent des indicateurs typographiques déclencheurs d'une citation directe (Mourad, 2000 : 497). A travers ces différentes interprétations, nous remarquons que ces signes graphiques ont plusieurs rôles et ne se limitent pas à la définition générale figurant dans les dictionnaires. Par suite, il nous a paru intéressant de creuser dans ce champ d'étude. Autrement dit, nous avons opté pour l'étude des guillemets dans le domaine littéraire afin de déceler ses différentes facettes. Et Le confident fut notre choix.

En fait, Le confident constitue le premier roman d'Hélène Grémillon, paru chez les Editions J.C Lattès en 2010. Réalisant un énorme succès (bestseller), il a été traduit en vingt-sept langues et a obtenu 5 prix littéraires à savoir :

Lauriers Verts de La Forêt des livres - Prix du premier roman 2010

Prix jeune talent littéraire des Clubs de lecture de Saint-Germain-en-Laye 2011

Prix Emmanuel-Roblès - Prix des lecteurs de la Ville de Blois 2011

Prix Montalembert « Coup de cœur du jury » 2011

Prix Palissy 2011 (source : site Prix littéraires)

Le récit, qui se déroule en 1972, relate l'histoire d'une éditrice, Camille, âgée de 35 ans, qui vient de perdre sa mère. Elle reçoit les lettres de condoléances et parmi celles-ci, elle trouve une grande enveloppe à l'intérieur de laquelle, un long courrier 
sans signature relatant l'histoire de jeunesse d'un certain Louis. Croyant d'abord que ce message lui est parvenu par erreur, elle reçoit chaque mardi un courrier de cette même personne anonyme qui poursuit la narration d'une histoire. Surprise, elle commence à se convaincre que c'est une méthode novatrice de la part d'un jeune auteur qui s'adresse à elle en tant qu'éditrice afin de publier son manuscrit.

A travers ces lettres, le roman tisse l'histoire de deux amours impossibles, de quatre destins brisés. Progressivement, en recoupant les passages, Camille se rend compte que cette longue missive la concerne et recèle un terrible secret sur sa propre vie. En fait, via une intrigue alambiquée, le lecteur garde le souffle vu le suspense du récit. En effet, sur fond de la Deuxième guerre mondiale, c'est le récit d'une jeune bourgeoise nommée Madame M. (Elisabeth) qui, souffrant d'un mal d'enfant, décide d'aller vivre loin de Paris à la province de Champagne, afin de fuir les regards de reproche de sa stérilité. En province, elle se lie d'amitié avec Annie, la fille d'un ouvrier, qui adore la peinture. L'amitié entre ces deux femmes va évoluer au point de conclure un marché abominable. Madame M. va prêter son mari à Annie afin de lui faire un enfant.

Au début, Paul, le mari de Madame M., refuse mais devant le désespoir et l'insistance de sa femme accepte cette folie à condition d'accomplir cet acte une seule fois. Or qui sème le vent, récolte la tempête. Annie a gagné le cœur de Paul et devient enceinte. Elle n'arrive pas à avouer à Louis, son amour d'enfance, cette machination diabolique. Après l'accouchement, Madame M. ne pense qu'à détruire la vie d'Annie pour l'entrainer au suicide et se protéger ainsi de toute tentative de récupérer l'enfant. Apparemment, Madame M. réussit son stratagème, et trente-cinq ans après, quand sa « fille » lui annonce qu'elle est enceinte, tous les remords de culpabilité resurgissent et elle se suicide en commettant un accident de voiture.

L'auteure garde le suspense jusqu'à la dernière page et le lecteur découvre que la vraie mère de Camille, "Annie », n'est pas morte. Ainsi, le récit met l'accent sur le côté psychologique des protagonistes, et ce à travers cinq voix principales qui relatent leurs confidences. Un chevauchement de polyphonie surprenant qui nécessite du talent dont a fait preuve Hélène Grémillon. En effet, le synopsis (1) représente l'intrigue romanesque:
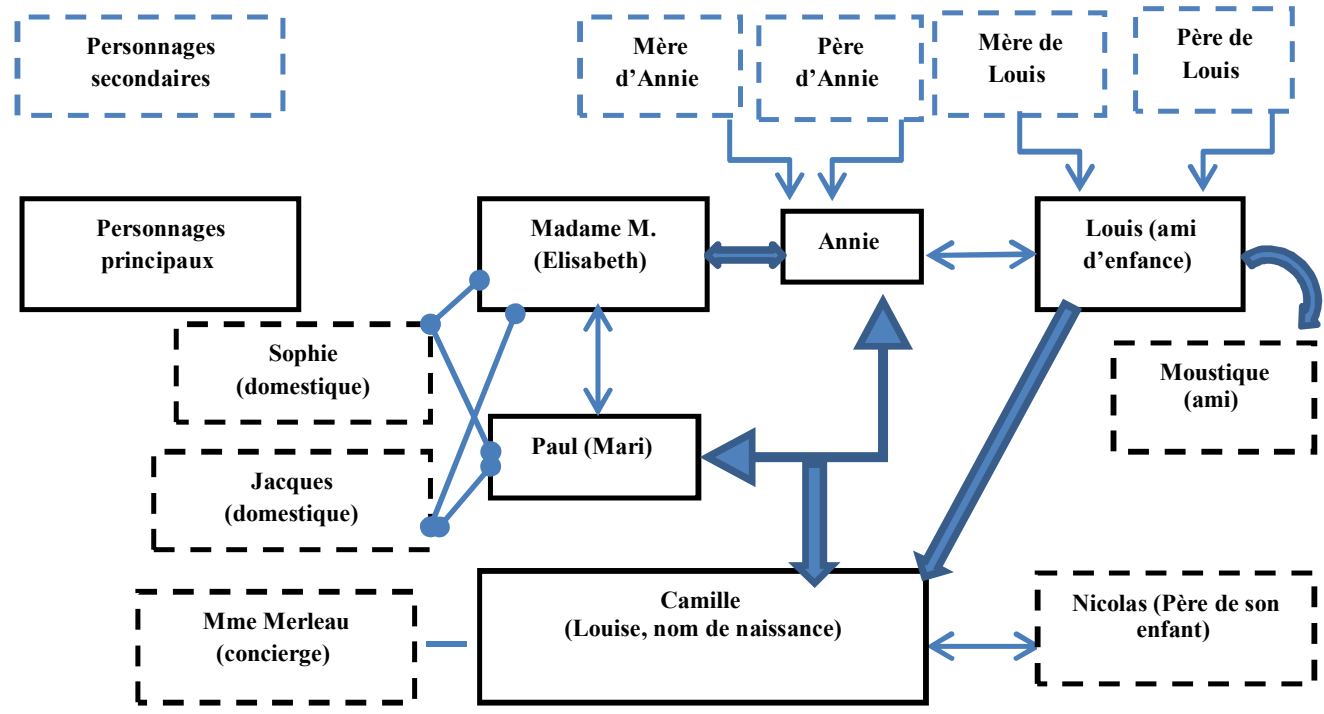

Synopsis (1): L'intrigue romanesque 


\section{Problématique}

A travers cette œuvre romanesque, et tout au long des différents récits enchâssés, nous remarquons la récurrence des guillemets. Récurrence, accompagnée par la diversité des syntagmes encadrés. Dans notre corpus, les guillemets sont exploités par Hélène Grémillon comme suit :

\begin{tabular}{|l|l|c|}
\hline \multirow{4}{*}{ Les guillemets } & $\begin{array}{c}\text { Nature de la séquence } \\
\text { encadrée }\end{array}$ & Récurrence \\
\cline { 2 - 3 } & Vocable/ mot isolé & 82 fois \\
\cline { 2 - 3 } & Syntagme/ groupe de mots & 91 fois \\
\cline { 2 - 3 } & Propositions & 25 fois \\
\cline { 2 - 3 } & Citations & 101 fois \\
\hline
\end{tabular}

Tableau illustratif des séquences encadrées par les guillemets

Par suite, cette récurrence des guillemets a donné naissance à une panoplie de questions à savoir : Pourquoi existe-t-il cette variété d'emploi ? La présence des guillemets est-elle un acte prémédité ou un simple symbole typographique secondaire? L'auteure désire-t-elle transmettre un message implicite via ces séquences guillemetées?

A cet égard, plusieurs travaux ont fait des guillemets leurs objets d'étude. Une taxinomie a été élaborée. «Parmi les guillemets autres que ceux qui introduisent le style direct, il semble que l'on puisse distinguer trois catégories : les guillemets linguistiques, les guillemets discursifs et les guillemets que j'appellerai énonciatifs.» (Vigneau-Rouayrenc, 1984 : 2). En fait, pour Vigneau-Rouayrenc (1984), les guillemets linguistiques encadrent des vocables isolés ou des syntagmes brefs qui constituent un écart à la norme. Ils connotent un niveau de langue qui n'est pas celui en usage d'ordinaire dans les textes littéraires. Par suite, elle distingue trois types de guillemets linguistiques à savoir les guillemets grammaticaux, lexicaux et idiolectaux. Quant aux guillemets discursifs, ils protègent la parole du narrateur de ce qui n'est pas elle. Ils constituent un moyen par lequel le scripteur signale un changement de voix. Enfin, les guillemets énonciatifs «ne signalent ni un changement de voix, ni une déviance quelconque par rapport à la norme prescriptive. Ils ne font qu'exprimer la relation du scripteur à l'énoncé et sont donc d'une utilisation tout à fait personnelle » (Vigneau-Rouayrenc, 1984 : 5). Ceux-ci comprennent les guillemets stylistiques et aléthiques.

En ayant cette typologie comme toile de fond, nous avons opté dans notre étude pour une approche séquentielle. En fait, celle-ci «s'attache à étudier la façon dont chaque énoncé s'insère dans son contexte » (Léon, 1999 : 20-21).

Il nous a paru intéressant d'approfondir notre recherche sur le rôle des guillemets dans Le confident. En effet, «placé entre ces signes doubles, un segment acquiert ainsi une visibilité qui permet d'indiquer son appartenance à un autre niveau discursif - en rupture avec l'espace situé hors-guillemets. » (Ponge, 2011 :399)

Par conséquent, notre problématique se base sur l'étude du rôle des guillemets. Autrement dit, nous allons décortiquer cette œuvre romanesque pour démontrer les différents emplois des guillemets et comment ils constituent un outil important au niveau de la polyphonie. En fait, nous allons analyser l'exploitation de ceux-ci par 
Grémillon et comment elle a procédé pour faire un recoupement entre les différents récits 《noncaux » et «toncaux » enchâssés de son œuvre. (Damourette et Pichon, 1949: 1703). En d'autres termes, comment Grémillon a varié l'emploi des guillemets pour le va-et-vient effectué entre les récits noncaux (relevant du temps présent) et les récits toncaux (relevant du passé). En effet, Grémillon débute son roman par un récit au temps présent, récit qui la transpose dans un temps passé plein de récits secondaires alambiqués. A travers cette subtilité le lecteur est amené à se concentrer afin de pouvoir suivre la trame romanesque. Le synopsis (2) présente les différents locuteurs et leurs destinataires dans ces récits emboités :

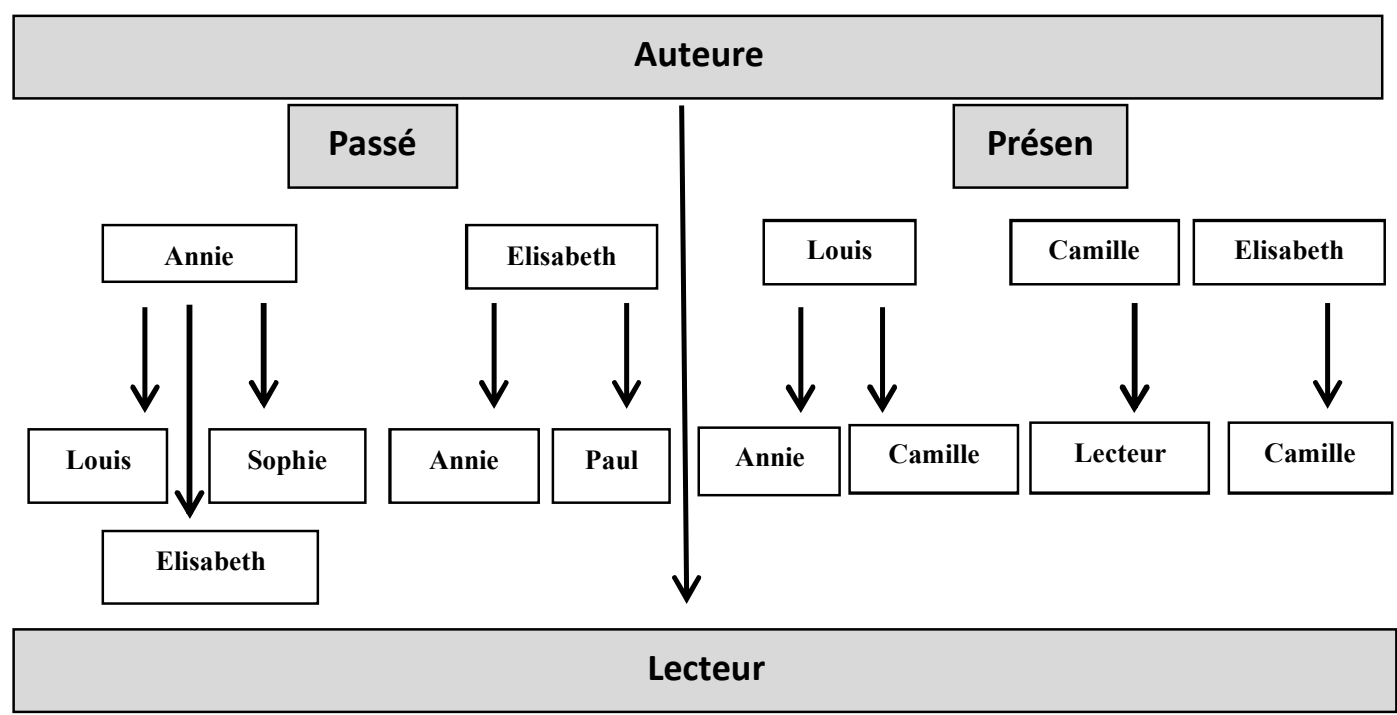

\section{Synopsis (2) : Les différents locuteurs et leurs destinataires dans l'intrigue}

\section{Développement}

Pour notre recherche, l'étude des guillemets est scindée en deux parties. La première partie porte sur l'étude des différentes fonctions des guillemets. Nous avons opté dans ce travail pour la taxinomie d'Augustyn concernant les vocables ou propositions guillemetés. Quant à la deuxième partie, elle se consacre à l'étude des îlots textuels.

\section{I.Première partie : les fonctions des guillemets}

D’après Augustyn les séquences encadrées par des guillemets jouissent de plusieurs fonctions à savoir :

«- la fonction purement autonymique

- la fonction citationnelle (citation autonome et non autonome)

- la fonction de dénomination

- la fonction de modalisation

- le proverbe » (Augustyn, $2015: 18$ )

Par suite, nous allons voir si ces différentes fonctions existent dans notre corpus ou au contraire, les séquences guillemetées sont dépourvues de signification. 


\section{a.La fonction autonymique :}

L'autonymie a été définie par plusieurs chercheurs. Delavigne voit qu'elle « fait porter l'attention sur les faits discursifs. Effaçant la transparence du langage, elle le densifie et le donne à voir, créant une focalisation sur les signes.» (Delavigne, 2013 : 2). Pour Jørgensen, elle y voit des signes « qui consistent en un changement de registre énonciatif.» (Jørgensen, 1999 :1). Idée approuvée par Maingueneau estimant que «l'énonciateur indique au lecteur que son discours ne coïncide pas avec luimême, sans en général en donner la raison. Pour la découvrir, pour interpréter les guillemets, le lecteur doit tenir compte du contexte et en particulier du genre du discours.» (Maingueneau, 2012 :184). Par conséquent, dans son récit «noncal», l'auteure a guillemeté certains vocables qui renvoient à d'autres énonciateurs, afin d'attirer l'attention du lecteur comme suit:

(1)- Que vous le vouliez ou non, votre père s'est illustré et vous devez prendre cette médaille.

-Mon père ne s'est pas «illustré », stupide grande gueule de travers, il s'est suicidé et il faut que vous le disiez à ma mère (Grémillon, p.12)

(2) Maman m'avait dit qu'elle me comprenait. Je lui avais dit qu'elle ferait une grand-mère merveilleuse. Elle m'avait répondu "sûrement». Et puis elle avait ajouté que c'était bien d'avoir un enfant, mais que c'était encore mieux de l'avoir à deux (Grémillon, p.94)

(3) Là encore, j'avais vraiment du mal à imaginer que mes parents ne m'aient jamais parlé de cette maison. Et puis j'avais fait des recherches sur un quelconque lieu-dit - «l'Escalier »-sans rien trouver. (Grémillon, p.158)

(4) Combien de gens se suicident ainsi dans des «accidents», pour éviter la culpabilité à leurs proches ?. (Grémillon, p.300)

A travers ces exemples, l'auteure exploite l'autonymie pour souligner une polyphonie de voix. Et cette polyphonie appelle le lecteur à un déchiffrement. Déchiffrement indispensable à la compréhension de l'intrigue, étant donné que les 4 vocables présents dans les exemples susmentionnés constituent les piliers de notre récit. Pour l'exemple (1), le vocable guillemeté renvoie au grand type qui présenta à Camille la médaille décernée à son père. Le lecteur s'informe ainsi de la mort du père (Paul) qui «s'est porté volontaire pour ouvrir la voie» (p.12) d'un champ miné. De même, il découvre l'étrange réaction de la jeune fille (Camille) face à l'annonce de la mort de son père. Ainsi cette autonymie attire l'attention du lecteur dès la deuxième page du roman au personnage du père qui représente le pilier principal de l'intrigue. C'est lui dont la femme était stérile, c'est lui qui va accepter d'avoir d'un enfant avec une autre femme et c'est lui qui va aimer la mère de son enfant. Et c'est cet amour qui déclenchera la jalousie de sa femme et ruinera la vie de tous les héros du récit.

Pour l'exemple (2), Grémillon change de registre et guillemète un vocable assumé par Elisabeth. La polyphonie souligne un tiraillement chez Elisabeth et cette idée est renforcée par le cotexte qui suit. En fait, le lecteur est capable d'interpréter la continuité de ce tiraillement dans l'exemple (4) où l'énonciateur dédouble son discours. En fait, le guillemetage du terme «accidents » représente un commentaire implicite de l'énonciateur qui désire se débarrasser de ce tiraillement. Par le biais de cette modalisation autonymique, l'énonciateur «parle tout en commentant en même 
temps sa parole en train de se faire. En parlant ainsi de sa propre parole, l'énonciateur produit une sorte de boucle dans son énonciation» (Maingueneau, 2012: 179-180).

Quant à l'exemple (3), il reprend le terme exprimé par Louis à savoir « l'Escalier». En fait, c'est le nom de la demeure qui constitue le foyer de notre intrigue. Cette polyphonie nous permet l'interprétation suivante : Grémillon exploite ce vocable en tant que terme "delphique ", expression empruntée à Hermans qui « appelle "termes delphiques" des termes qui ont une apparence familière, mais dont l'interprétation se démarque de l'interprétation qui en est faite habituellement » (Hermans, 1997 : 272). En fait, l'escalier dans son emploi usuel signifie un moyen soit pour monter soit pour descendre. Dans notre cas, cette maison devait être un lieu de gradation et de changement de classe étant donné qu'Annie (de classe moyenne) va fréquenter la classe bourgeoise. Cependant, l'idée diabolique d'Elisabeth a transformé cette demeure en un lieu de dégradation, car les vies de toutes les personnes ont été gâchées. Idée mise en relief via :

La typographie (guillemets, majuscule et traits d'union)

Le cotexte qui précède (un quelconque lieu-dit).

\section{b. La fonction citationnelle (citation autonome et non autonome) :}

A travers le discours citant, figurent des discours cités encadrés par des guillemets. Ces discours cités par d'autres locuteurs représentent le discours direct (désormais DD). « Le locuteur à la source de cet énoncé doit donc être explicitement mis en scène, i.e. il doit être clairement identifiable par des indices linguistiques (nom propre, syntagme nominal), même si ce n'est pas dans la même phrase.» (Augustyn, 2015 :18). En effet, le discours direct traditionnel est constitué d'un verbe introducteur en incipit suivi de deux points puis d'une citation guillemetée selon la formule et les exemples suivants :

\section{Sujet $\mathrm{X}+$ verbe introducteur $\quad \longrightarrow \quad$ : + « ----------- »}

(5) J'ai repensé au «Depuis qu'elle te connait, Madame M.va de mieux en mieux » de Sophie et au « Si j'ai accepté qu'on s'installe ici, c'est pour que tu ailles mieux » de son mari. (Grémillon, p.54)

(1 er « je » Annie / discours citant $)-(2 \mathrm{e} « \mathrm{je} »:$ Paul / discours cité $)$

(6) «Je l'ai tricotée en me souvenant de comment ça fait quand je te serre dans mes bras ». J'avais laissé maman me serrer fort dans ses bras, moi qui ne la laissais plus m'approcher. (Grémillon, p.78)

(1 er « je » mère d'Annie / discours cité) - (2e « je » : Annie / discours citant)

D'après Augustyn, « en se basant sur des critères énonciatifs et syntaxiques, on distinguera la citation autonome et la citation non autonome.» (Augustyn, 2015 : 19). Selon elle, l'emploi citationnel est autonome en cas d'autonomie énonciative ou syntaxique. L'autonomie énonciative implique un je renvoyant au locuteur du discours cité et non du discours citant (exemples 5 et 6 ).

Cependant pour l'autonomie syntaxique, Augustyn estime que c'est en général « une phrase mais aussi une phrase averbale ou une réponse elliptique. Ce type est 
souvent marqué par l'usage de : «...», mais ce n'est pas toujours le cas, car le verbe introducteur peut être en incise ou après le passage entre guillemets, et il peut ne pas y en avoir.» (Augustyn, 2015 : 19). Cette autonomie syntaxique figure dans notre corpus comme suit :

(7) Jacques était resté à L'Escalier. «Pour l'entretenir jusqu'à notre retour », disait-elle. (Grémillon, p.87)

(8) Sans lui répondre son mari était sorti du salon. Il avait l'air bouleversé, il était même passé devant moi en me prenant pour Sophie : «Vous n'avez donc rien à faire dans cette maison, vous ? » (Grémillon, p.52)

(9) « Tu veux voir ce que ça fait d'être loin, eh bien, tu vas voir, ne compte pas sur nous pour t'écrire ». Il m'avait lancé ça, juste après m'avoir offert le chevalet. (Grémillon, p.86)

Quant aux emplois citationnels non autonomes, ils «sont intégrés énonciativement et syntaxiquement dans le discours enchâssant» (Augustyn, 2015 : 20). Selon elle, les citations non autonomes peuvent être :

du discours indirect introduit par verbe + que :

(10) Et je me plaisais à l'imaginer dans ses dîners en ville répéter que « non, elle ne ressentait rien » (Grémillon, p.88)

\section{b- des îlots citationnels :}

Augustyn définit les îlots citationnels comme étant une séquence guillemetée plus petite que la phrase et «intégrée syntaxiquement au discours citant.» (Augustyn, 2015: 20)

(11) A cet instant-là, je me souviens avoir pensé qu'elle ne me trouvait plus du tout « agréable à aimer » comme elle disait. (Grémillon, p.57)

(12) Mon père n'a jamais murmuré à ma mère qu'on pouvait « mourir d'amour » (Grémillon, p.156)

des formes mixtes du type : selon $\mathrm{X}$, d'après $\mathrm{X} \ldots$...

(13) A la radio, il venait d'entendre le discours d'investiture de Reynaud: «Vaincre c'est tout sauver, succomber c'est tout perdre » (Grémillon, p.240)

(14) Aux prédictions des astrologues qui assuraient que selon les horoscopes de MM.Hitler et Mussolini, il n'y aurait «pas de guerre cet été », je préférais lire qu'on avait observé, dans l'est de la France et en Allemagne, le passage de nombreux Jaseurs de Bohême, comme en 1870, comme en 1914 : ces oiseaux, dont les plumes se terminent par une sorte de boule de cire rouge sang, avaient la réputation d'annoncer de grandes catastrophes. (Grémillon, pp.217-218) 


\section{c. La fonction de dénomination}

Les guillemets jouissent d'une fonction de dénomination «lorsque leur usage signale un acte de dénomination, l'assignation d'une étiquette dénominative. L'accent est donc mis à la fois sur le signifiant et le signifié » (Augustyn, 2015 : 20). Selon elle, il peut s'agir d'une catégorisation, d'un concept, d'un néologisme ou d'un titre.

(15) Elle n'aurait pas pris le risque que j'écrive quelque chose qui nous trahisse. Elle ne me le disait pas, mais je le savais. Je l'appelais «la Giraudoux » de mon courrier. (Grémillon, p.18).

(16) Sophie ne me servait que des aliments dits « recommandés »: roquette, céleri, artichauts, asperges, truffes... (Grémillon, p.175)

Il est à noter que pour l'exemple (15), l'auteure a explicité cette dénomination par la note explicative suivante en bas de page (« Du 29 juillet 1939 au 21 mars 1940, Jean Giraudoux fut en charge de la censure dans les médias français »). Quant à la l'exemple (16), la dénomination est confirmée par le cotexte qui précède à savoir le participe passé (dits) attribué au substantif (aliments).

\section{d. La fonction de modalisation}

Pour ce qui est de la fonction de modalisation, Augustyn voit que les guillemets recèlent cette fonction «quand ils signalent une distance de l'auteur vis-à-vis du terme : l'auteur montre qu'il ne prend pas complètement en charge ce terme. On peut trouver plusieurs cas de figure:

-soit le terme est inapproprié par rapport à ce qu'il désigne :

- soit le terme n'est pas tout à fait satisfaisant vis-à-vis du lecteur (le terme est trop savant, ou au contraire trop familier) :

- soit l'auteur se refuse à prendre en charge le terme car une telle prise en charge est contraire à (ou éloignée de) ses convictions, son point de vue.

Cette fonction est parfois accompagnée des gloses méta-énonciatives (si je puis dire, comme on dit, passez-moi l'expression...). » (Augustyn, 2015 : 21)

(17) Je buvais de ce vin prétendument «aphrodisiaque», je conjuguais l'aphrodisiaque à toutes les sauces, les confitures, les sirops, c'était pathétique. (Grémillon, p.176)

(18) Manifestement, ce libraire était au fait de la question. En le suivant à travers les rayonnages, je fus pris d'un fol espoir. Dans quelques secondes, je tiendrais l'« ouvrage de référence » entre mes mains, datés certes, mais « ouvrage de référence » quand même. (Grémillon, p.174)

(19) « Salauds! », « Voyous !», « Vauriens ! », ce sont d'abord les insultes des passants qui ont attiré mon attention, ils s'en donnaient à cœur joie contre un groupe de prisonniers agglutinés de l'autre côté de la rue, en face du café Piémont. (Grémillon, p.251)

A travers, l'exemple (17), le cotexte qui précède le vocable guillemeté (prétendument) constitue une glose de l'énonciateur, soulignant ainsi une certaine distance vis-à-vis du terme employé. Et ce refus de prise en charge du terme est concrétisé par le guillemetage ainsi que l'emploi de l'adverbe «pathétique ». 
Cependant pour l'exemple (18), le recours au raisonnement via le connecteur « mais » crée la modalisation. En fait, le « mais » introduit un revirement argumentatif dans l'énoncé. Autrement dit, l'idée introduite par le « mais » est contraire à celle abordée en incipit. La forme canonique de l'emploi du « mais » est selon l'équation (1) :

\begin{tabular}{|c|c|c|}
\hline $\begin{array}{l}\text { "X» } \\
\text { (Idée A) }\end{array}$ & + mais & $\begin{array}{l}\rightarrow \text { «Y » } \\
\text { (Réfutation de l'idée A) }\end{array}$ \\
\hline
\end{tabular}

\section{Equation (1)}

Or, nous découvrons que l'énonciateur a exploité le « mais » selon l'équation (2) :

\begin{tabular}{|c|c|c|c|}
\hline $\begin{array}{l}\text { «X»+ certes, } \\
\text { (Idée } A \text { ) }\end{array}$ & + mais & $\rightarrow$ & $\begin{array}{l}\text { «X» quand même } \\
\text { (Acceptation de l'idée } A \text { ) }\end{array}$ \\
\hline
\end{tabular}

\section{Equation (2)}

D'où le remplacement du revirement argumentatif par une approbation Ainsi, nous pouvons dire le terme « ouvrage de référence » précédant le « mais » est une autonymie désignant la voix de la collectivité, cependant le terme «ouvrage de référence » suivant le «mais » est une modalisation où l'énonciateur refuse de prendre en charge ce terme contraire à ses convictions, entrainant ainsi une ironie. La preuve en est le recours à la glose «quand même». En effet, " l'énonciateur emploie une expression et la montre en quelque sorte du doigt, indiquant par là qu'il ne l'assume pas vraiment; dans le cas de l'ironie, il produit un énoncé qu'il invalide en même temps qu'il parle » (Maingueneau, $2012: 200$ )

Quant à l'exemple (19), l'énonciateur se distancie des insultes émises par les passants. D'où l'emploi du guillemetage.

e.Le proverbe

Quant au proverbe, « il est considéré comme un cas particulier. Il se rapproche d'une citation avec une source collective qui ne peut être identifiée. C'est le cas des proverbes et des adages par exemple, qui appartiennent à tout le monde et à personne, ou encore du discours de la doxa, de l'opinion commune. Comme la source n'est pas réellement identifiable, contrairement aux citations, et que c'est une catégorie un peu trop particulière de modalisation, ils sont intégrés dans une catégorie spécifique.» (Augustyn, $2015: 22$ )

Dans notre corpus, figure une sentence passée en proverbe:

(20) «En octobre tonnerre, vendanges prospères » : diction de ce 4 octobre 1943. Je feuilletai distraitement le calendrier pour voir ce que les jours à venir nous préparaient. (Grémillon, p.70)

Ainsi à travers cette première partie, nous avons essayé de relever les différentes fonctions des séquences guillemetées dans notre œuvre romanesque, selon la taxinomie d'Augustyn. Il s'avère que l'auteure a exploité avec conscience ces différentes fonctions, faisant preuve d'une maitrise de la trame narrative. Le choix des différents guillemetages recèle des messages implicites nécessitant une interprétation de la part du lecteur. 


\section{II.Deuxième partie : les îlots textuels}

L'étude des différentes fonctions nous amènent à creuser davantage dans l'analyse des séquences guillemetées et ce au niveau des îlots textuels. En fait, « l'îlot textuel est un segment guillemeté, relevant de la modalisation autonymique. Mais il a la particularité d'apparaître au sein d'un discours indirect ou d'une modalisation en discours second sur le contenu, c'est-à dire au sein d'un discours représenté plus étendu relevant, lui, du mode standard.» (Fleck, 2015 : 2)

Autrement dit, les guillemets «marquent l'hétérogénéité scripturale : ils permettent au locuteur de signaler implicitement qu'il parle avec les mots des Autres. » (Bors, 2004 : 229).

A cet égard, il s'avère nécessaire de mentionner la typologie proposée par $\mathrm{J}$. AUTHIER-REVUZ (1992 : 41-42) qui répartit en trois catégories les diverses formes de discours rapporté : formes marquées et univoques (discours direct, discours indirect, modalisation en discours second), formes marquées exigeant un travail interprétatif (segments relevant d'une modalisation autonymique marqués par des guillemets, par des italiques ou par une certaine intonation) et formes purement interprétatives (discours direct libre, discours indirect libre, citations cachées, allusions).

Par conséquent, en se basant sur les travaux d'Authier-Revuz, Kjersti Fløttum (2001) s'est approfondie sur l'étude des îlots textuels figurant dans les trois cas :

« (I) îlot textuel en DI (Jean dit que ... "X" ...)

(IIa) îlot textuel hors DI avec source explicite (selon (les paroles de) Jean, ... "X"

(IIb) îlot textuel hors DI sans source explicite (... "X"...)» (Fløttum, 2001:3)

Pour ce qui est de notre corpus, Grémillon a tissé ces trois cas avec excellence comme suit :

(21) Sophie est venue me prévenir que Paris était « ville ouverte » (Grémillon, p.253) (Cas : I)

(22) Quand nous étions encore « les inséparables », comme disait mon père. (Grémillon, p.125) (Cas : IIa)

(23) Ils en seraient sûrement venus aux mains si les fameux «putains de stukas » ne nous avaient pas foncé dessus. (Grémillon, p.122) (Cas : IIb)

Ainsi, en (21), Grémillon met en exergue le recoupement de voix via un îlot textuel dans un discours indirect. Autrement dit, nous avons la voix d'Annie et de Sophie à travers la narration d'Annie. Cependant, en (22) et (23) se manifeste l'enchâssement des voix dans une narration hors discours indirect : Louis et son père dans (22) et Louis et le «on» indexical de collectivité en (23) qui marque une distanciation de la part du locuteur.

Ces exemples illustrent bien la polyphonie des voix narratives dans notre œuvre romanesque. Tout au long des différents récits narrés par différents personnages, le lecteur se trouve face à plusieurs genres de locuteurs. A cet égard, Fløttum (2001 : 9) distingue «deux catégories principales d'êtres discursifs : locuteurs virtuels et non-locuteurs». Selon elle, Les locuteurs virtuels (LV) sont des images de personnages susceptibles de prendre la parole. Parmi les LV, il y a le locuteur de l'énoncé (1), le locuteur textuel (L), l'allocutaire de l'énoncé (a), l'allocutaire textuel 
(A) et les troisièmes ( $3 \mathrm{e})$. Ces derniers sont ceux qui peuvent être indiqués par les pronoms de la troisième personne ou des syntagmes nominaux. De même, Fløttum estime la présence d'une variante spécifique de $\mathrm{LV}$, à savoir le locuteur représenté (LR), responsable d'un discours représenté (ou rapporté).

Le confident offre au lecteur une variété de locuteurs virtuels. Nous sommes en présence de différents locuteurs de l'énoncé à savoir Louis, Annie, Elisabeth et Camille. Chacun d'eux relate son récit tout en ayant recours à des îlots textuels guillemetés soulignant d'autres voix afin:

de les mettre en relief pour attirer l'attention du lecteur,

d'attribuer leur responsabilité à leurs locuteurs respectifs

de se distancier de ces propos pour supposer la présence de deux points de vue (désormais pdv). Et ce comme suit :

(24) Visiblement fébrile, elle n'arrêtait pas de remuer sa cuillère dans sa tasse. «Ne compare pas ce qui ne se compare pas ». Annie avait lentement remonté son chandail sur les épaules. (Grémillon, pp.44-45)

(Locuteur de l'énoncé (1) : Louis)

(Locuteur textuel (L) «»: Annie)

(pdv1: on compare ce qui ne se compare pas \# pdv2: réfutation de pdv1)

(25) «La pièce sans murs ». Elle l'appelait comme ça parce que les murs disparaissaient sous un immense miroir et sous de lourdes tentures rouges. (Grémillon, p.49)

(1: Annie)

(L « »: Elisabeth/Madame M.)

(pdv1: la pièce a des murs. \# pdv2 : réservation vis-à-vis de l'expression d'Elisabeth)

(26) « Tes parents vont bien ». Madame M. me transmettait toujours cette phrase. Toute souriante. «Tes parents vont bien ». Sale menteuse. (Grémillon, p.87)

(1: Annie)

(L « »: Elisabeth)

(pdv1: les parents ne vont pas bien (réalité) \# pdv2 : les parents vont bien "mensonge")

(27) Je sais, c'était stupide, mais « les erreurs naissent souvent des certitudes », Paul le répétait assez. (Grémillon, p.187)

(1 : Elisabeth)

(L « »: Paul)

(pdv1 : les erreurs ne naissent pas de certitudes \# pdv2 : réservation vis-à-vis de pdv1)

(28) J'ai essuyé quelques « non » polis et réservés. (Grémillon, p.39)

(1: Camille)

(L « »: des inconnus)

(pdv1 : attente d'une réponse positive \# pdv2 : réponse négative) 
Quant aux non-locuteurs (NL), « ce sont des êtres discursifs responsables d'un point de vue (pdv) sans pour autant pouvoir prendre la parole ou produire une énonciation. La voix publique ou l'opinion générale, le $\mathrm{ON}$, est un tel non-locuteur, n'ayant pas la propriété de pouvoir prendre la parole directement.» (Fløttum, 2001:9)

(29) «Madame M. s'ennuie toute seule », avançaient les uns, « et puis elle est encore si jeune », surenchérissaient les autres. Le village entier essayait de trouver des raisons à cette amitié contre nature entre cette bourgeoise de grande famille et leur petite Annie. (Grémillon, p.43)

(NL : les gens du village)

(1: Louis, non-responsabilité totale du guillemetage ---- NL: 3e personne responsable)

(30) Alors que la porte du restaurant se refermait derrière nous, les haut-parleurs des voitures de police crachaient dans la rue. "Allô, allô, toutes les personnes trouvées dans la rue après minuit seront conduites au poste et gardées jusqu'à 5 heures du matin » (Grémillon, p.124)

(NL : police)

(1: Louis, non-responsabilité totale ---- NL : $3 \mathrm{e}$ personne responsable)

(31) En Allemagne, le ministre Goebbels avait sonné l'hallali de cette terrible chasse aux Juifs et ces ordures nazies avaient cassé tellement de vitrines et de vaisselles qu'ils appelaient ça la « Nuit de cristal » (Grémillon, p.168)

(NL : les nazis)

(1: Elisabeth, non responsabilité totale du guillemetage --- NL : 3e personne responsable)

(32) Je ne supportais plus la campagne de natalité qui faisait rage, c'étaient partout les mêmes imprécations. «Faites des enfants ! Faites des enfants, il faut réparer les pertes de $1914 »$ (Grémillon, p.169)

(NL : journaux représentant la voix des journalistes)

(1: Elisabeth, non responsabilité totale du guillemetage --- NL : 3e personne responsable)

(33) C'était la course à la natalité. Les journaux allemands exhibaient des exemples à suivre : "Schumann était un petit cinquième, Bach avait sept frères et sœurs, Haendel neuf, Durer seize, Wagner était le cadet de huit enfants, Mozart de dix... » (Grémillon, p.214)

(NL: journaux représentant la voix des journalistes)

(1: Elisabeth, non responsabilité totale du guillemetage --- NL : 3e personne responsable)

A travers les exemples (29 à 33), nous remarquons que Grémillon a inséré des non-locuteurs dans son intrigue. Et elle ne s'est pas contentée de recourir à des nonlocuteurs de même nature. En fait, elle a créé une variation au niveau de cette catégorie et nous découvrons que ces non-locuteurs englobent des policiers, des nazis et des journalistes. Par suite cette variété vise à transformer ce récit fictif en un monde quasi-réel. 


\section{Conclusion :}

En guise de conclusion, Le confident représente une œuvre romanesque faisant preuve de la finesse du style narratif. Hélène Grémillon a tissé une intrigue constituée de maints récits «noncaux » et « toncaux » qui se recoupent, emportant le lecteur dans un voyage entre le présent et le passé. La présence des différents énonciateurs pour ces différents récits a créé un enchâssement alambiqué aiguisant la curiosité du lecteur.

L'auteure était consciente de l'importance des guillemets au niveau énonciatif et elle les a maniés avec maitrise. En fait, elle a exploité les 5 fonctions des guillemets à savoir la fonction autonymique, la fonction citationnelle, la fonction de dénomination, la fonction de modalisation et le proverbe.

A travers la fonction autonymique, Grémillon a exploité le changement de registre énonciatif. Quant à la fonction citationnelle, elle a renvoyé la responsabilité des énoncés au locuteur du discours cité et non du discours citant tout en variant entre les citations autonomes et non autonomes. Pour ce qui est de la fonction dénominative, Grémillon a focalisé le signifiant et le signifié. D'autre part, elle s'est distanciée vis-à-vis de certains vocables via la fonction de modalisation, et ce afin de ne pas en assumer la responsabilité. Et elle a même eu recours au proverbe qui constitue un cas particulier de modalisation étant donné que sa source «n'est pas réellement identifiable » selon Augustyn.

D'autre part, Grémillon a travaillé sur la notion des îlots textuels via les guillemets au niveau de son discours rapporté. En fait, nous sommes en présence d'îlots textuels en discours indirect, d'îlots textuels hors discours indirect avec source explicite et d'îlots textuels hors discours indirect sans source explicite. Et par le biais de ces différentes facettes des séquences guillemetées, l'auteure a entrainé le lecteur dans le labyrinthe des locuteurs virtuels et des non-locuteurs et ce afin de renforcer l'idée de la polyphonie des voix dans son récit fictif.

De ce qui précède, nous pouvons conclure que les guillemets constituent un outil primordial au niveau énonciatif, un moyen efficace de distanciation et de prise de responsabilité, et un moyen de va-et-vient entre les différents locuteurs discursifs. Loin d'être symbole typographique secondaire, les guillemets ont un rôle central au niveau de la narration. Reste à savoir si les guillemets jouissent de la même importance ou pas dans d'autres disciplines. Question qui ouvre la voie vers de nouvelles pistes de recherche. 


\section{Références bibliographiques :}

Corpus:

GREMILLON, Hélène (2013); Le confident, Folio, Paris.

Ouvrages :

AUGUSTYN, Magdalena (2015); Annotations des marques de la subjectivité langagière: discours rapporté, passages entre guillemets et lexique des affects ; Manuel de codage Octobre 2015 ; LIDILEM - Université Stendhal Grenoble 3, [Consultation en ligne:

http://lidilem.u-grenoble3.fr/IMG/pdf/manuel_de_codage_dr_peg.pdf, 26/8/2017]

AUTHIER-REVUZ, Jacqueline, 1992, « Repères dans le champ du discours rapporté», in L'Information grammaticale, volume 55, pp38-42. [Consultation en ligne:

$1 / 6 / 2017]$

http://www.persee.fr/doc/igram_0222-9838_1992_num_55_1_3186,

AUTHIER-REVUZ, J. (1998) « Le guillemet : un signe de langue écrite à part entière», in: Defays, J.M., Rosier, L. \& Tilkin, F. (éds), A qui appartient la ponctuation?, Actes du colloque international et interdisciplinaire de Liège, 13-15 mars 1997. Paris : Duculot, pp. 373-388.

BORS, Edit (2004) ; "Pour une approche polyphonique du journal intime» in Verbum VI/1, pp. 229.240, Akadémiai Kiadó, Budapest, 2004. [Consultation en ligne: https://btk.ppke.hu/uploads/articles/8528/file/6-1-18.pdf, 3/9/2017]

DAMOURETTE, J.; PICHON, E., (1949), «Des mots à la pensée. Essai de grammaire de la langue française 1911-1940», I-VII, D’Artrey, Paris.

DELAVIGNE, Valérie (2013) ; Le double jeu de l'autonymie, Le fait autonymique dans les langues et les discours, 2000, Paris, France. 2002. [Consultation en ligne: https://hal.archives-ouvertes.fr/hal-00919527 Submitted on 17 Dec 2013, 2/6/2017]

FLECK, Frédérique (2015), « La possibilité d'un îlot. La répétition comme indice de littéralité » in De Lingua Latina 11, Revue de linguistique en ligne du Centre Alfred Ernout, Université Paris Sorbonne [Consultation en ligne:http://www.parissorbonne.fr/IMG/pdf/DLL_11_F-Fleck.pdf, 7/8/2017]

FLØTTUM, Kjersti (2001); «Fragments guillemetés dans une perspective polyphonique », [Consultation en ligne: http://www.hum.au.dk/romansk/polyfoni/Diverse\%20artikler/Flottum_guillemets.ht m 3/7/2017]

HERMANS A. (1997); " Mots et termes en sociologie », in Boisson C. et Thoiron P., Autour de la dénomination, Lyon, Presses universitaires de Lyon, p. 269-278. 
JØRGENSEN, Kathrine Sørensen Ravn (2000), Etude stylistique du discours de «l'autre» dans Madame Bovary, [Consultation en ligne:

http://C:/Documents\%20and\%20Settings/Owner/My\%20Documents/Downloads/24 07-744-1-PB\%20(2).pdf 3/7/2017]

LEON, Jacqueline (1999); «Les entretiens publics en France, analyse conversationnelle et prosodique », CNRS EDITIONS, Paris.

MAINGUENEAU, Dominique (2012) ; «Analyser les textes de communication » Armand Colin, Paris.

MOURAD, G (2000) ; Présentation de connaissances linguistiques pour le repérage et l'extraction de citations. In TALN, 7ème Conférence Annuelle sur le Traitement Automatique des Langues Naturelles ; pp. 495-501; Lausanne, Suisse 16 - 18 octobre 2000.

PONGE, Myriam (2011), «Le guillemetage : une illustration par la ponctuation de l'enrichissement mutuel des codes oral/écrit (espagnol, français) », in Colloque international "Les rapports entre l'oral et l'écrit dans les langues romanes", Dec 2011, Paris / Saint-Denis, France. pp.397-409, 2012, [Consultation en ligne: https://hal-univ-paris8.archives-ouvertes.fr/hal-01345468, submitted on 30/7/2016, 3/9/2017]

ROBERT, Paul (2011) ; «Le Petit Robert », Nouvelle édition millésime.

VIGNEAU-ROUAYRENC, Catherine; Les guillemets ou l'énonciation non-verbale chez L-F Céline et H. Poulaille. In: L'Information Grammaticale, N. 20, 1984. pp. 11-17. Doi:10.3406/igram.1984.2265 [Consultation en ligne: http://www.persee.fr/doc/igram_0222-9838_1984_num_20_1_2265, 20/8/2017]

Sitographie :

Site : Prix littéraires

http://www.prix-

litteraires.net/detail_prix_auteur.php?auteur=4906_Helene_Gremillon 


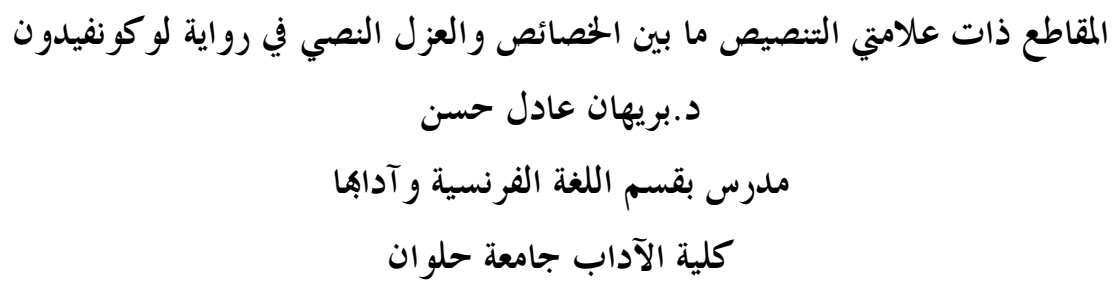

الملتخص:

تمثل علامتا التنصيص أداة هامة على مستوى النص المكتوب. وبعيداً عن كوفما علامة شكلية،

تقوم علامتا النصيص بدور هام في النصوص الأدبية. ومن خلال الرواية الأدبية لوكونفيدون وهي أول عمل أدبي للروائية إيلين جريميون، سنقوم بتحليل المقاطع ذات علامتي التنصيص من حيث الوظائف والعزل النصي. 